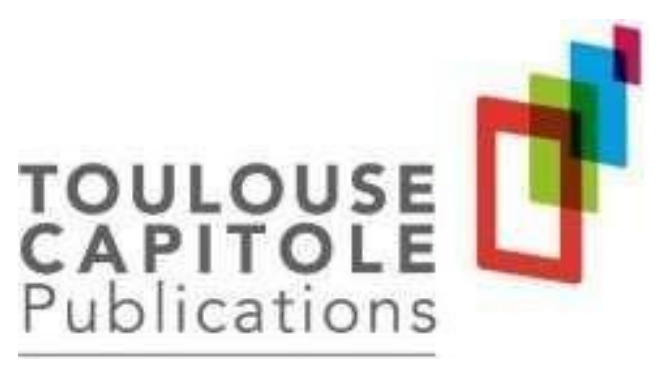

« Toulouse Capitole Publications » est l'archive institutionnelle de l'Université Toulouse 1 Capitole.

\title{
La justice américaine et les cultures génétiquement modifiées
}

\author{
MASTOR WANDA
}

Référence de publication: MASTOR (W.), «La justice américaine et les cultures génétiquement modifiées », Revue juridique de l'environnement, 2012, n 1, p. 89-98.

Pour toute question sur Toulouse Capitole Publications, contacter portail- publi@ut-capitole.fr 


\section{La justice américaine et les cultures génétiquement modifiées}

\section{Résumé}

Symbole de l'agriculture saisie par la chimie au nom d'objectifs affichés qui en dissimulent d'autres, Monsanto est au coeur de nombreuses batailles judiciaires à travers le monde. L'arrêt Monsanto Co. Et al. V. Geertson Seed farms et al., rendu par la Cour suprême des Etats-Unis le 21 juin 2010, semble à la fois satisfaire et décevoir chaque partie. Monsanto s'en félicite presque avec arrogance, quand d'autres évoquent au contraire un «revers » que la plus haute juridiction des Etats-Unis lui aurait infligé. Le juge de district de San Francisco, Jeffrey White, n'a pas hésité à suspendre une culture de betteraves, puis à en permettre son fauchage. De son côté, le juge Charles Breyer, également juge de la cour de San Francisco, est à l'origine de l'affaire des luzernes qui va remonter jusqu'à la Cour suprême. La comparaison de l'ensemble de ces décisions permet de confronter l'audace des juges du fond à l'ambivalence de la plus haute juridiction des Etats-Unis.

\section{Abstract}

Symbol of agriculture seized by chemistry in the name of the stated objectives which conceal others, Monsanto is at the heart of many legal disputes around the world. The Monsanto Co. et al. V. Geertson Seed Farms et al. decision, delivered by the Supreme Court of the United States on June 21, 2010 seems both to satisfy and disappoint each party. Monsanto welcomes it almost arrogantly, while others suggest on the contrary, a "setback" that the highest court of the United States would have inflicted. District Judge Jeffrey White of San Francisco did not hesitate to stop a beetroot growing, and then allow its mowing. For his part, Judge Charles Breyer, also a judge of the Court of San Francisco, is the source of the alfalfa case which is going up to the Supreme Court. The comparison of all these decisions allows to compare the audacity of the lower courts to the ambivalence of the highest court of the United States.

« Producing more. Conserving more. Improving lives. That's sustainable agriculture and that's what Monsanto is all about ». La vidéo disponible sur la page d'accueil du site internet de Monsanto est une véritable ode à un monde meilleur. Y défilent des paysages somptueux sur fond de musique douce et de commentaires, via un timbre profond, sur le lien entre la culture de la terre et le développement. Aucun continent n'est oublié, chaque slogan est pesé (la lutte contre la pauvreté, le soutien des agriculteurs, le développement économique des pays pauvres, le droit à l'éducation...) dont d'aucuns n'oseraient dire qu'ils ne les partagent pas. Plus qu'une excellente stratégie de communication, la très utopique page d'accueil de la société Monsanto est aux antipodes de la mauvaise presse qu'elle a auprès des défenseurs de l'environnement. Ce royaume fondé par John Francis Queeny, originellement inventeur de produits chimiques, est associé à l'agent orange qui a causé tant de désastres irréversibles, aux conséquences toujours visibles, au Vietnam. Il est aujourd'hui l'un des premiers vendeurs de pesticides dans le monde, et l'un des principaux producteurs de semences génétiquement modifiées (coton, soja, luzerne, maïs, colza).

Symbole de l'agriculture saisie par la chimie au nom d'objectifs affichés qui en dissimulent 
d'autres, Monsanto est au cœur de nombreuses batailles judiciaires à travers le monde. Joutes qui opposent la plupart du temps des petits agriculteurs à la multinationale, et dont l'affaire canadienne qui s'est terminée par un arrêt de la Cour suprême rendu quatre voix contre cinq serait le zénith ${ }^{1}$. La Cour constitutionnelle fédérale allemande a ainsi, dans un arrêt du 24 novembre $2010^{2}$, réaffirmé la nécessité pour les lois d'établir un principe de précaution en ce qui concerne les OGM. Une plainte avait été déposée par un Land contre une loi fédérale qui obligeait un apiculteur cultivant des plantes génétiquement modifiées à verser réparation en cas de contamination, par transport de pollen, d'une récolte voisine. On connaît l'attachement, notamment pour des raisons historiques, du Tribunal de Karlsruhe pour les principes liés au droit à la vie et à la dignité humaine en général. Rappelant dans cette espèce que le génie génétique modifie la structure même de la vie, il énonce strictement qu' " étant donné que l'état des connaissances des conséquences à long terme de la dissémination dans l'environnement des modifications génétiques ne fait pas l'objet de recherches approfondies, le législateur a un devoir spécial de protection ».

Cette décision fut suivie d'un arrêt de la Cour de justice de l'Union européenne qui a pris également sa source en Allemagne ${ }^{3}$. Une question préjudicielle fut posée par un juge allemand à propos de l'interprétation du règlement $\mathrm{n}^{\circ} 1829 / 2003$ organisant l'autorisation et la surveillance des denrées alimentaires et des aliments pour animaux génétiquement modifiés ainsi que leur étiquetage. Le litige opposait des apiculteurs au Freistaat Bayern, propriétaire de terrains sur lesquels étaient cultivés du maïs de l'entreprise Monsanto, à propos de la présence, dans les produits apicoles, de pollens de maïs génétiquement modifiés. La première question posée à la Cour de Luxembourg était la suivante : le pollen issu d'un maïs génétiquement modifié peut-il être lui-même considéré comme une structure génétiquement modifiée ? Monsanto avait obtenu au niveau communautaire une autorisation de mise sur le marché du maïs génétiquement modifié (le « maïs MON 810 »), culture par ailleurs interdite en Allemagne. La Cour rappelle qu'en vertu du quatrième considérant de la directive $n^{\circ}$ 2001/18/CE, " les organismes vivants disséminés dans l'environnement, en grande ou en petite quantité, à des fins expérimentales ou en tant que produits commerciaux, peuvent se reproduire dans l'environnement et franchir les frontières nationales, affectant ainsi d'autres États membres. Une telle dissémination peut produire des effets irréversibles sur l'environnement ». Par ailleurs, il découle du deuxième considérant du règlement $\mathrm{n}^{\circ} 1829 / 2003$ sur l'autorisation et la surveillance des denrées alimentaires et des aliments pour animaux génétiquement modifiés ainsi que leur étiquetage souligne un niveau élevé de protection de la vie et de la santé humaine dans l'exécution des politiques communautaires.

La Cour a répondu par la négative à la première question posée, estimant que le pollen présent dans le miel ou utilisé comme complément alimentaire n'était pas un « OGM » au sens du règlement $n^{\circ}$ 1829/2003, « dès lors qu'il ne posséderait plus, au moment où il est incorporé au miel ou est destiné à l'alimentation, notamment sous forme de complément alimentaire, aucune aptitude concrète et individuelle à la reproduction et que la seule présence d'ADN et/ou de protéines transgéniques ne serait pas suffisante à cet égard ». Néanmoins, et la satisfaction des apiculteurs réside dans ce second point, la Cour a affirmé que des produits comme du miel contenant un tel pollen contenaient des « ingrédients » produits à partir d'OGM au sens du règlement. En conséquence, le pollen relève du champ d'application du règlement et doit être soumis au régime d'autorisation.

Dans un arrêt en date du 6 juillet de la même année ${ }^{4}$, la Cour de justice avait déjà estimé que Monsanto ne pouvait interdire la commercialisation dans l'Union européenne de la farine de soja argentine contenant une séquence ADN pour- tant brevetée par elle. La multinationale a ainsi l'habitude des 
prétoires ; il est particulièrement intéressant d'observer le sort récent que lui réserve la justice du pays dans lequel elle a son siège.

L'écologie est une question à laquelle les Etats-Unis paraissent historiquement - voire culturellement - peu sensibles. Ils n'ont pourtant pas été épargnés par les catastrophes écologiques aux conséquences dévastatrices. Qu'elles soient naturelles, tel le Dust Bowl dans les années trente, au cœur des Raisins de la colère de John Steinbeck, ou d'origine humaine, telle la marée noire en Alaska provoquée en 1989 par le pétrolier Exxon Valdez, dont la Cour suprême a rédigé une déconcertante conclusion le 25 juin $2008^{5}$. La plus grande démocratie du monde est également la plus grande agriculture. Le maïs et le soja sont, à plus de $90 \%$, des OGM, dont $80 \%$ pour le premier et $93 \%$ pour le second proviennent de Monsanto. Pourtant, y compris outre-Atlantique, des voix se sont élevées pour dénoncer la forme et le fond de ce monopole. Il n'est pas caricatural d'affirmer que les juges des États sont souvent plus progressistes que ceux de Washington. Les décisions des juridictions qu'en France nous qualifierions de première instance ou d'appel sont suivies avec attention, et les parties retiennent souvent leur souffle avant que la Cour suprême ne se prononce en dernier ressort. Les procès environnementaux se multiplient, et la plus haute juridiction s'en saisit progressivement ${ }^{6}$. Mais les défenseurs de l'environnement ont été plus d'une fois déçus jusqu'à présent. Dans l'affaire Exxon citée plus haut, comme dans celle relative aux émissions de gaz à effet de serre du 20 juin $2011^{7}$, la Cour de Washington a été moins généreuse que les juges du fond. Dans cette dernière, elle avait à juger le recours intenté par huit États contre cinq industriels de l'électricité (dont l'American Electric Power Company). Alors que la cour d'appel de l'Etat de New York avait estimé que la loi sur les «nuisances publiques » rendait légitime la plainte des Etats et de plusieurs associations, la Cour suprême, à l'unanimité ${ }^{8}$, l'a précisément rejetée dans un arrêt rendu le 21 juin 2011. Celle-là même qui, le 2 avril 2007, avait infligé un revers à l'inaction de l'administration contre le réchauffement climatique en jugeant que les émissions de gaz à effet de serre étaient bien « polluants » 9. Mais l'arrêt n'avait été rendu qu'à cinq voix contre quatre et induisait surtout la nécessité d'une règlementation. Dans l'arrêt rendu en 2011, la Cour, cette fois unanime, ne prit finalement pas position, et renvoya au Congrès et à l'Agence américaine de l'environnement (EPA) le soin de réguler la question des émissions, de se saisir de la question du réchauffement climatique. Posture finalement logique du point de vue la séparation des pouvoirs, mais peu satisfaisante pour les écologistes qui savent bien que la Cour peut être plus activiste quand elle le souhaite...

En matière d'OGM, le scénario est identique pour la luzerne, en suspens pour la betterave à sucres. L'arrêt Monsanto Co. Et al. V. Geertson Seed farms et al. rendu par la Cour suprême le 21 juin 201010 semble à la fois satisfaire et décevoir chaque partie. Monsanto s'en félicite presque avec arrogance, quand d'autres évoquent au contraire un « revers » que la plus haute juridiction des Etats-Unis lui aurait infligé... A l'audace des juges du fond (I) succède l'ambivalence de la Cour suprême (II).

\section{I- L'AUDACE DES JUGES DU FOND}

La lutte contre la surpuissance de Monsanto a plusieurs visages. Celui des petits agriculteurs, qui, revêtus de l'insouciance de David face à Goliath, n'ont pas hésité à déclencher des procédures judiciaires. Celui des défenseurs de l'environnement, des célèbres associations écologistes à l'auteur d'un documentaire remarqué à l'intitulé évocateur : « Le monde selon Monsanto - de la dioxine aux OGM, une multinationale qui vous veut du bien » ${ }^{11}$. Mais ce visage, c'est également ceux des juges du district nord de Californie. Également connu pour avoir été au cœur de l'affaire Wikileaks, le juge de district de San Francisco Jeffrey White n'a pas hésité à suspendre une culture de betteraves par une décision du 13 août 2010, puis à en 
permettre son fauchage (B). Le juge Charles Breyer, également juge de la Cour de San Francisco, est à l'origine de l'affaire des luzernes qui remontera jusqu'à la Cour suprême et qui obligera son frère, Stephen Breyer, à se déporter $(\mathrm{A})$.

\section{A- LE CAS DES LUZERNES TRANSGÉNIQUES}

Tout part d'une plainte déposée en février 2006 par plusieurs associations, dont le Center for food safety, et d'entreprises de production de semences contre le ministère de l'Agriculture (l'USDA) et l'agence de protection de l'environnement (l'EPA). Le recours était dirigé contre une autorisation du 25 juin 2005 de commercialisation de luzernes (alfafa) transgéniques, tolérantes à l'herbicide RoundUp Ready produit par Monsanto. Selon les plaignants, cette autorisation « arbi- traire et capricieuse ${ }^{12}$ faisait fi des dommages causés à l'environnement et n'avait pas été précédée d'une étude d'impact. De ce fait, elle violait tant la loi sur la protection de l'environnement (The National Environmental Policy Act) que celle sur les procédures administratives (Administrative Procedure Act).

En conséquence, les plaignants demandaient notamment au juge de constater que le ministère de l'Agriculture violait lesdites lois, de déclarer que l'autorisation de commercialisation pouvait avoir des effets très significatifs sur l'environnement et d'enjoindre au ministère d'élaborer une étude d'impact.

Le juge Charles Breyer, de la Cour de district de San Francisco, rend sa décision le 13 février 2007 13. La question centrale était finalement la suivante : l'autorisation de commercialisation de luzernes transgéniques a-t-elle un « impact significatif » sur l'environnement ? Le ministère de l'Agriculture, à travers l'agence de santé animale et végétale (the Animal and Plant Health Inspection Service [ci- après APHIS]), a estimé dans un premier temps que quiconque désirait introduire de la luzerne génétiquement modifiée devait obtenir une autorisation préalable. En mai 2003, l'entreprise Monsanto a demandé à l'APHIS de considérer que la luzerne produite par elle ne présentait pas un risque pour l'environnement, et par conséquent ne nécessitait pas une réglementation. Le juge Breyer rap- pelle que l'APHIS avait trois possibilités face à cette requête : l'accepter totale- ment, partiellement (en considérant que la luzerne tolérante au RoundUp ne présentait pas un risque significatif dans certaines zones géographiques, un risque significatif dans d'autres) ou la rejeter. Pour prendre sa décision, l'agence a conduit une étude d'impact (Environmental Assessment [EA]) et procédé à des auditions. La majorité des personnes interrogées s'opposèrent à une déréglementation, notamment en raison du risque de la contamination des cultures de luzernes biologiques par les abeilles. A l'argument écologique se greffait un argument commercial : $75 \%$ de l'ensemble des exportations de luzernes concernent le Japon, qui n'autorise pas l'importation de luzerne tolérante au glyphosate.

Malgré ces résultats, l'APHIS décida de donner pleine satisfaction à Monsanto en juin 2005, lui délivrant une sorte de certificat d'absence d'impact significatif (Finding of no significant impact [FONSI]), contesté par les plaignants devant le juge de San Francisco. S'appuyant sur la jurisprudence antérieure, le juge Breyer rappelle dans les motifs de la décision qu'il lui appartient de vérifier l'absence de caractère « significatif » du risque, celui-ci devant s'apprécier tant au regard du contexte que de l'intensité. Pour les requérants, ce qu'ils nomment la «contamination biologique » a des effets négatifs économiques et socioéconomiques sur les agriculteurs (et par conséquent, sur le choix des consommateurs), qui sont incapables de maintenir une agriculture biologique dans ce contexte.

La Cour, à travers la voix de Charles Breyer, considère que les requérants ont réussi à établir une 
«probabilité raisonnable » que leurs cultures biologiques seraient contaminées par celles transgéniques. Elle conclut que tous les arguments avancés par l'APHIS n'étaient pas « convaincants », celle-ci n'ayant pas réussi à démontrer qu'elle avait exercé un contrôle strict (hard look) sur l'impact de la déréglementation sur l'environnement. Appel fut interjeté, et la Cour suprême des Etats-Unis finit par se saisir de l'affaire et rendit une décision le 21 juin 2010.

\section{B- LE CAS DE LA BETTERAVE TRANSGÉNIQUE}

La décision dont il va être ici question fut rendue le 13 août 2010 par la même cour que précédemment, à travers cette fois la voix du juge Jeffrey White ${ }^{14}$. Les faits sont similaires à ceux de l'affaire de la luzerne : le 17 mars 2005, l'APHIS a délivré une autorisation commerciale pour la betterave H7-1 des entreprises Monsanto. Plusieurs associations et agriculteurs biologiques décidèrent de porter plainte, estimant que les effets d'une telle autorisation sur l'environnement avaient insuffisamment été évalués au préalable. Selon le juge White, les plaignants ont réussi à mettre en évidence les « erreurs »de l'APHIS, qui ne sont «ni mineures ni insignifiantes ». Il regrette que les défendeurs mettent en avant l'argument économique des conséquences d'un retrait de l'autorisation de commercialisation des betteraves, au regard de l'importance de la potentialité des effets néfastes sur l'environnement. En conséquence, il suspend l'autorisation en attente d'une nouvelle étude d'impact, dont le projet n'a été publié que le 11 octobre $2011^{15}$. Au moment où nous écrivons, l'appel du jugement est pendant devant la neuvième cour de circuit. Comme pour la luzerne, l'APHIS pro- pose trois alternatives dans son étude provisoire : une commercialisation possible, interdite, ou partielle. L'Agence se donne soixante jours à partir du 14 octobre pour procéder à des auditions et rendre sa décision. Le dénouement final devrait avoir lieu aux alentours de mai 2012.

Nouvel épisode de ce feuilleton judiciaire, le juge White a ordonné, le 30 novembre $2010{ }^{16}$, la destruction de certaines parcelles de betteraves génétiquement modifiées, arguant des impacts irréversibles sur l'environnement. Le 25 février 2011, la cour d'appel a cassé l'arrêt de la cour de district

${ }^{17}$, rappelant, à travers la voix du juge Sidney Thomas, que le juge White n'avait pas été capable de prouver que lesdites plantations présentaient des risques irréversibles pour l'environnement. Dans un style quasi épique, n'hésitant pas à citer le Français Olivier de Serres, " le père de l'agriculture », Richard II de Shakespeare ou Jurassic Park de Michael Crichton, la cour d'appel conclut sévèrement que «l'évocation de la théorie du chaos ne suffit pas à légitimer » l'ordre de destruction du juge White. Cet arrêt revêt même des allures de réprimandes à l'égard d'un juge capricieux qui n'aurait pas conscience des retombées économiques de son acte « faucheur».

Sans attendre l'étude d'impact, ni même son ébauche, le ministère de l'Agriculture a de nouveau autorisé la betterave à sucre tolérante au RounUp Ready le 4 février 2011. Il s'agit d'une autorisation partielle, en attendant la publication des résultats de l'évaluation des impacts environnementaux. Plusieurs associations, dont le Center for food safety ont de nouveau contesté l'autorisation devant le juge White, mais le débat s'est déplacé sur le terrain de la délocalisation judiciaire entre la cour du district de Californie et celle de Columbia ${ }^{18}$.

La décision du 13 août 2010 n'est finalement qu'une demi-victoire pour les requérants, qui espéraient que la Cour non seulement suspende les cultures des betteraves génétiquement modifiées, mais interdise également la vente de celles déjà récoltées. Or, le juge White a décidé que les cultures plantées avant le 13 août, date du jugement, pouvaient être récoltées : « la Cour va limiter la suspension (the 
vacatur) à toutes les betteraves à sucre génétiquement modifiées plantées après la date de cette décision (...). La Cour n'enjoindra pas la destruction des cultures génétiquement modifiées déjà plantées ${ }^{19}$.

Bien que représentant une avancée pour les anti-OGM, il ne faut pas pour autant conclure que « la justice américaine », contrairement à ce qu'a titré Le Monde.fr, « interdit la betterave à sucre transgénique ${ }^{20}$. Non seulement l'affirmation est inexacte, mais pourrait bien être rendue obsolète par l'étude d'impact qui ne sera sans doute pas publiée avant le printemps 2012. Une « demi-victoire », en l'occurrence pour les deux parties, est l'expression qui convient également à l'arrêt de la Cour suprême des Etats-Unis rendu le 21 juin 2010.

\section{II- L'AMBIVALENCE DE LA COUR SUPRÊME}

Chaque décision de la Cour suprême, sur un sujet relatif aux grandes questions sociétales, est un évènement. Traduction cursive différente oblige, posture interprétative incomparable, le contenu est si substantiel qu'il peut receler des ambiguïtés dont chaque partie se saisit. Si la solution ne laisse guère de place au doute, les motifs sont plus complexes et peuvent être interprétés dans un sens ou un autre. L'ambiguïté ne provient d'ailleurs pas tant des formulations de la juridiction que de leur interprétation par les destinataires.

Cette ambivalence dans la réception d'une décision de justice est particulière- ment évidente dans l'exemple de la décision Monsanto Co. Et al. V. Geertson Seed farms et al., la multinationale s'en félicitant autant que ses adversaires. Pendant que sur son site internet, Monsanto annonce que « la Cour suprême tranche en faveur des agriculteurs » (sous-entendu, ceux cultivant la luzerne tolérante au RoundUp ${ }^{21}$, l'une des parties plaignantes évoque « une défaite » pour l'industriel ${ }^{22}$... De leur côté, les medias français ont largement interprété la décision comme « une victoire de Monsanto devant la Cour suprême ${ }^{23}$. Le lecteur éprouve alors une certaine gêne à la lecture des commentaires qui fleurissent sur internet, ne sachant plus qui est le puissant et le misérable, et surtout qui des deux finit par l'emporter. Il

se souvient alors de la morale des « animaux malades de la peste » de la Fontaine ${ }^{24}$ et peut céder à la facilité de croire que la victoire est fatalement du côté du puissant. L'analyse de la source primaire, à travers le seul prisme de la volonté de la compréhension, sans parti-pris idéo- logique, permet d'en saisir le sens (A) et la portée (B).

\section{A- UN SENS CERTAIN}

En juin 2008, le ministère de l'Agriculture, Forage Genetics International, Monsanto et plusieurs agriculteurs utilisant de la luzerne RoundUp Ready ont fait appel de la décision du juge Breyer. A deux voix contre une, la Ninth Circuit Court of Appeals a confirmé la décision de première instance en maintenant l'injonction ${ }^{25}$. A travers la voix du juge Schroeder, la cour d'appel a estimé que la Cour de district avait correctement effectué le contrôle de proportionnalité, et suspendu à raison l'autorisation de commercialisation. L'opinion dissidente du juge Randy Smith ne concerne qu'un aspect procédural. Comme les requérants, il estime qu'avant de prononcer l'injonction, la Cour inférieure aurait dû procéder à des auditions de témoins.

L'affaire parvient à la cour de Washington par la voie du certiorari. Le progressiste juge Stephen Breyer, frère de l'auteur de la première décision du 13 février 2007, s'est autorécusé pour cette unique 
raison familiale. Le conflit d'intérêt semblait pourtant plus évident pour le juge Clarence Thomas, qui fut l'un des avocats de Monsanto entre 1976 et 1979.

Du point de vue de la solution, la décision est claire et sans ambiguïté : les juges suprêmes annulent l'injonction de la suspension de la vente de semence de luzerne génétiquement modifiée. Décision d'autant plus univoque qu'elle n'est pas accompagnée d'opinions concordantes (mais d'une opinion dissidente néanmoins), et d'autant plus légitime aux yeux de ses partisans qu'elle est adoptée à une large majorité. A travers la voix du juge Alito, la Cour estime que les juges du fond ont agi de façon «prématurée », l'étude d'impact n'ayant pas été publiée à l'époque des décisions - et toujours pas à l'heure où nous écrivons. La plus haute des juridictions condamne en quelque sorte le «zèle » du juge californien en soulignant qu'il a outrepassé ses prérogatives : "In sum, the District Court abused its discretion in enjoining APHIS from effecting a partial deregulation and in prohibiting the possibility of planting in accordance with the terms of such a deregulation ».

Les dirigeants de Monsanto se sont félicités de cette décision, annonçant non sans malice leur confiance dans le futur rapport du ministère de l'Agriculture : « Tous les agriculteurs peuvent faire confiance à l'expertise de l'USDA (ministère de l'Agriculture), et être convaincus que les autorisations futures des cultures biotechnologiques doivent s'appuyer maintenant sur des faits scientifiques, pas sur des spéculations » ${ }^{26}$. Mais en réalité, quel que soit le point de vue que le lecteur adopte a priori, la décision est bien plus complexe qu'elle n'y paraît.

\section{B- UNE PORTÉE INCERTAINE}

A la victoire proclamée par Monsanto à l'issue du délibéré, les détracteurs opposent sa défaite. Les motifs de la décision ne sont pas aussi manichéens, et les arguments interprétatifs des deux parties sont somme toute valables. Du point de vue de la solution, Monsanto a réussi à faire censurer les décisions des juges du fond. Très concrètement, l'injonction du juge de la cour de district « ne tient pas », celui-ci ayant outrepassé sa fonction. Mais les motifs de la décision lais- sent entrevoir une lueur d'espoir pour les anti-OGM. Ce que la Cour remet catégoriquement en cause, c'est l'injonction de l'interdiction de la commercialisation de la luzerne Monsanto. Mais la Cour suprême ne se prononce pas sur le fond, sur le fait de savoir si cette culture est légale ou non. Sa posture n'est d'ailleurs pas sans rappeler celle de l'affaire Exxon : à aucun moment, dans cet arrêt du 25 juin 2008, la Cour ne revient sur les événements du 24 mars 1989. Sans faire allusion à l'ampleur de la marée noire, au pessimisme des études scientifiques qui ont suivi, les juges majoritaires se limitent à l'étude la question suivante : le droit maritime envisage-t-il les dommages punitifs ? Il en va un peu de même dans la présente affaire, la Cour s'interdisant d'entrer dans le débat de fond relatif aux effets des cultures transgéniques sur l'écologie en général et sur les cultures bio voisines en particulier.

L'interdiction de semer perdure donc, tant que l'APHIS n'a pas publié son étude d'impact sur l'environnement. D'ici là, une seule certitude doit animer la justice fédérale : c'est au Gouvernement et non à elle, notamment à travers l'Agence de santé animale et végétale, qu'il appartient d'établir des conclusions scientifiques : "Until such time as the agency decides whether and how to exercise its regulatory authority, however, the courts have no cause to intervene ».

Il est regrettable que les adversaires de Monsanto n'aient pas utilisé à leur profit l'opinion dissidente du juge Stevens ${ }^{27}$. Le juge libéral, qui a l'habitude de cosigner des opinions avec le juge Breyer, 
absent du délibéré, commence par relever que ses collègues majoritaires ont mal interprété la décision de la cour de district. Le dissident poursuit en rappelant les données extrêmement délicates de l'affaire, les positions radicalement opposées des forces en présence, pour conclure que la Cour a fait « du mieux qu'elle pouvait ». En l'absence de l'étude d'impact, la seule solution était celle de l'attente, concrétisée par l'injonction de l'interdiction de la commercialisation. Selon le juge Stevens, la Cour suprême aurait dû confirmer la décision de la Cour de district pour au moins deux raisons : parce que, d'une part, la juridiction de San Francisco avait appliqué correcte- ment la loi ; et parce qu'elle avait, d'autre part, apporté la seule réponse raison- nable aux risques potentiels de la luzerne génétiquement modifiée. La position du juge du fond n'était donc ni « prématurée », ni zélée.

Jusqu'à la publication de ce rapport aux alentours du printemps 2012, personne n'est en réalité en mesure de crier victoire. Les deux parties se comportent comme deux équipes adverses dans un match où l'arbitre a besoin de recul pour prendre sa décision. Pendant ce temps de réflexion, chaque partie applaudit des deux mains, faisant ainsi pression sur les décideurs. Il en va de même avec les décisions de justice américaines en matière d'OGM. Quand la décision n'est pas parfaitement claire, à l'instar de celle de la Cour suprême, chaque partie annonce à grands coups de communiqués de presse la défaite de l'autre.

Pour ne pas faire baisser la cote des actions, pour séduire l'opinion publique, pour influencer le Gouvernement. Il n'est pas certain que le consommateur anti- OGM y gagne quelque chose. Il doit de toute façon encore attendre l'épilogue de cette affaire, en se félicitant que la justice américaine, historiquement peu sensible aux questions environnementales, ait fini par s'en saisir. C'est toujours ainsi qu'ont commencé les grandes batailles judiciaires et que des victoires incontestables ont été emportées.

\section{Notes de bas de page}

1. Cour suprême du Canada, Monsanto Canada Inc. v. Schmeiser, [2004] 1 S.C.R. 902, 2004 SCC 34.

2. BVerfG, $\quad 1 \quad$ BvF $\quad 2 / 05 \quad$ vom $\quad$ 24.11.2010,
http://www.bverfg.de/entscheidungen/fs20101124_1bvf000205.html

3. Affaire C-442/09 Karl Heinz Bablok e.a./Freistaat Bayern.

4. Affaire C-428/08 Monsanto technology LLC/Cefetra BV e.a.

5. $\quad$ Exxon Shipping Co. Et Al. v. Baker et Al, 554 US 471 (2008).

6. Les arrêts dont il sera ici question ont d'ailleurs tous été rendus par la voie du Certiorari, la Cour suprême s'autosaisissant des affaires d'importance.

7. American electric power Co. Inc., et al v. Connecticut et al., 564 US (2011).

8. A huit voix précisément, la juge Sottomayor s'étant déportée pour avoir été partie à cette affaire en tant que juge fédéral.

9. Massachusetts et al. V. Environmental protection agency et al., 549 US 497 (2007). La Cour fut une nouvelle fois divisée entre libéraux (les juges Stevens, Ginsburg, Souter et Breyer, le juge Kennedy ayant joué son rôle classique de « bascule ») et conservateurs (les juges Roberts, Scalia, Thomas et Alito). 10. 549 US 497 (2010).

11. Documentaire réalisé par Marie-Monique Robin, coproduit par Arte France, Image et Compagnie, Productions Thalie, l'Office national du film du Canada et WDR, diffusé pour la première fois sur Arte le 1er mars 2008.

12. Complaint Alfafa, 2-15-2006, p. 2. Nous n'avons travaillé qu'à partir des sources directes ; tant les recours des plaignants que les décisions des juges de district sont disponibles sur le site de la Cour de 
district nord de Californie, United States District Court Northern District of California, http://www.cand.uscourts.gov/home

13. Geertson Seed Farms v. Johanns, 2007 WL 518624 (N.D. Cal. Feb. 13, 2007).

14. The United States Court for the Northern District of California, Center for food Safety et al. v. Thomas

J. Vilsack et al., no C 08-00484 JSW.

15. http://www.aphis.usda.gov/biotechnology/sugarbeet.shtml

16. Center for Food Safety v. Vilsack, no C10-04038 JSW (ND Cal. 2010).

17. Center for food safety v. Monsanto Company, 2011 US App. no 10-17719 (9th Cir. Feb. 25, 2011).

Les décisions de cette cour d'appel ont été consultées sur le site http://www.ca9.uscourts.gov

18. The United States Court for the Northern District of California, Center for food Safety et al. v. Thomas J. Vilsack et al., no C 11-00831 JSW du 17 mars 2011.

19. The United States Court for the Northern District of California, Center for food Safety et al. v. Thomas J. Vilsack et al., no C 08-00484 JSW, précitée, p. 9 (traduction par nos soins).

20. http://www.lemonde.fr/ameriques/article/2010/08/15/la-justice-americaine-interdit-la-betteravea- sucre-transgenique_1399159_3222.html

21. http://monsanto.mediaroom.com/index.php?s=43\&item $=855$

22. Andrew Kimbrell, " Supreme Court case a defeat for Monsanto's ambitions ", http://www.huffingtonpost. com/andrew-kimbrell/supreme-court-case-a-defe_b_620087.html

23. Pour ne reprendre que l'expression du Monde.fr, http://www.lemonde.fr/ameriques/article/2010/06/21/victoire-de-monsanto-devant-la-cour-supreme-desetats-unis_1376507_3222.html

24. «Selon que vous soyez puissant ou misérable, les jugements de cour vous rendront blanc ou noir $\gg$.

25. Geertson Seed Farms v. Johanns, 2008 US App. LEXIS 18752 (9th Cir. Sept. 2, 2008).

26. Voir le communiqué de presse sur le site de Monsanto, http://monsanto.mediaroom.com/index. php?s=43\&item $=855$

27. Sur les sites internet des opposants classiques à Monsanto, nous n'avons trouvé aucune mention de cette opinion dissidente, pourtant substantielle et fort bien argumentée. 